\title{
DE SÉbastien Roch À L'AgNeAu CHASTE OU DE LA CONSTRUCTION À LA DÉCONSTRUCTION DE LA MATRICE ROMANESQUE DE LA PÉDOPHILIE
}

\author{
Przemysław SzCZUR \\ Université Pédagogique, Cracovie
}

\begin{abstract}
Riassunto (It): L'obiettivo del presente articolo è di paragonare l'immagine della pedofilia in due romanzi : Sébastien Roch di Octave Mirbeau e L'agneau chaste di Franck Varjac. Mentre il primo ha contribuito a costruire la matrice romanzesca della pedofilia, il secondo prova a decostruirla.
\end{abstract}

Parole chiave (It): Pedofilia ; Romanzo francese ; Octave Mirbeau ; Franck Varjac

Mots-clés (Fr): Pédophilie ; Roman français ; Octave Mirbeau ; Franck Varjac

À la mémoire de ma grand-mère Rozalia (1931-2015)

\section{Introduction}

Dans La Volonté de savoir, Michel Foucault a placé la «pédagogisation du sexe de l'enfant» parmi les «grands ensembles stratégiques, qui développent à propos du sexe des dispositifs spécifiques de savoir et de pouvoir » (FOUCAULT, 1976 : 137-138) à partir du siècle des Lumières. Cette «pédagogisation », qui se manifeste par une surveillance active de tout ce qui touche à l'activité sexuelle des enfants, devenue sujet de vives inquiétudes et préoccupations des parents et éducateurs, paraît avoir gagné en intensité entre les $\mathrm{XIX}^{\mathrm{e}}$ et $\mathrm{XXI}^{\mathrm{e}}$ siècles. La carrière et l'évolution sémantique du mot «pédophilie » qui, au XIX ${ }^{\mathrm{e}}$, signifiait « amour des enfants, sans implication sexuelle » (COUROUVE, 1985: 179) et la psychiatrisation du phénomène qu'il désigne aujourd'hui (cf. CHAUMON, 2005), semblent en témoigner. Si encore dans les années 1970 la pédophilie a pu faire partie du mouvement de libération sexuelle, ce qui a aussi trouvé une traduction au niveau littéraire (cf. WOLF, 2014 : 199-210), elle semble aujourd'hui l'un des sujets qui focalisent le plus les peurs liées à la sexualité. Gert Hekma a, entre autres, finement analysé les raisons de ce phénomène (cf. HEKMA, 2010 : 357359), je ne reviendrai donc pas là-dessus. Je voudrais me pencher sur les conséquences littéraires de cette situation sociale, en postulant l'existence d'une «matrice romanesque » propre au thème pédophile. J'emploie ce terme pour renvoyer à un modèle discursif, créé dans le cadre du roman du XIX ${ }^{\mathrm{e}}$ siècle, mais dont on retrouve selon moi des traces dans le discours social contemporain sur la sexualité intergénérationnelle. Je commence mon analyse par un examen de Sébastien Roch d'Octave Mirbeau (1890) qui, à mon avis, fut à l'origine de la matrice précitée. Je me penche ensuite sur la façon dont le mini-roman de Franck Varjac, intitulé L'Agneau chaste, publié en 2000, et continuant, dans une certaine mesure, la tradition du «roman pédophile ${ }^{1}$ » de l'après Mai 68, déconstruit cette même matrice. Bien que les protagonistes des romans de Mirbeau et Varjac aient tous les deux plus de treize ans et soient plutôt des adolescents que des enfants, les

${ }^{1}$ L'expression est de Nelly Wolf (WoLF, 2014 : 202).

Echo des études romanes XI/2, 2015 - 135 -

ISSN: 1804-8358 (Online) 
interprétations contemporaines des deux oeuvres témoignent de leur réception en tant que livres traitant de la pédophilie ${ }^{2}$. Cette dernière paraît en effet avoir acquis une acception très large, recouvrant différentes formes de sexualité intergénérationnelle, qu'elle soit basée sur la contrainte ou non et qu'elle implique des enfants ou des adolescents.

\section{Matrice romanesque de la pédophilie dans Sébastien Roch}

Paru en 1890, Sébastien Roch d'Octave Mirbeau me semble avoir joué un rôle de premier plan dans l'élaboration de la matrice romanesque de la pédophilie. Laurent Ferron parle de ce roman comme d' « un premier témoignage littéraire » sur le traumatisme d'un enfant violé (FERRON, 2001). En écrivant son livre, le romancier a suivi une tendance générale de l'époque car, comme le note AnneClaude Ambroise-Rendu : «(...) la fin du XIX ${ }^{\mathrm{e}}$ siècle est (...) marquée par l'essor assez considérable des dénonciations d'attentats à la pudeur sur enfants » (AMBROISE-RENDU, $2003: 31$ ). La chercheuse précise que la percée de ce sujet dans la presse a eu lieu dans les années 1880, du point de vue temporel, le roman de Mirbeau couronne donc cette décennie charnière et, dans la perspective sociale, il s'inscrit dans un phénomène socio-discursif qui dépasse largement la sphère littéraire. Influencé sans doute par ses propres expériences (cf. MICHEL, 2005 : 6667), mais aussi, très probablement, par la déferlante médiatique signalée par AnneClaude Ambroise-Rendu, Mirbeau construit la matrice romanesque de la pédophilie autour d'un couple de personnages dont les origines remontent au moins jusqu'au roman noir (gothique) du XVIII ${ }^{\mathrm{e}}$ siècle (cf. Ż ABSKI, $2006: 457-$ 458 ; BeAUMARCHAIS ; COUTY ; REY, 1984 : 1987-1988). Ce couple est composé d'un bourreau et de sa victime. Cette dernière a ici la particularité d'être un enfant, s'inscrivant dans une tradition qui dépasse le roman noir, et embrasse des courants aussi différents que le romantisme social (pour ne mentionner que Cosette dans Les Misérables de Victor Hugo) et le naturalisme (p.ex. la petite Lalie Bijard dans L'Assommoir d'Émile Zola). Les rapports entre ces deux personnages, en l'occurrence : le héros éponyme, adolescent, et son maître d'étude, un prêtre, le Père de Kern, sont construits à travers la thématique de la persécution, pour culminer dans l'épisode du viol du garçon par son éducateur. Comme dans nombre de romans noirs, p.ex. chez le marquis de Sade, dans Sébastien Roch, le thème de la sexualité, en l'occurrence celle du clergé, est relié à celui du mal. Dans la narration, les portraits du Père de Kern fonctionnent comme autant de prolepses inquiétantes annonçant cet événement clé de l'intrigue que constitue le viol. Y apparaît notamment le thème du regard persécuteur (cf. MIRBEAU, 1890 : 158$159,171)$ qui structure d'emblée les relations entre les protagonistes selon une

\footnotetext{
${ }^{2}$ L'âge du protagoniste de L'Agneau chaste fait que le terme d' «éphébophilie » (attrait pour les adolescents) semblerait plus approprié, mais les propos de Josyane Savigneau que je cite dans la suite de mon étude confirment la réception du livre comme roman sur la pédophilie. Quant au livre de Mirbeau, Pierre Michel l'analyse dans le contexte des scandales pédophiles dans l'Église catholique (Michel, 2005: 66, 237), bien que dans ce cas également il s'agisse plutôt d'« éphébophilie » dans la mesure où au moment d'être séduit par un prêtre le héros a quatorze ou quinze ans, comme l'a calculé Laurent Ferron (FERRON, 2001).
} 
logique de la contrainte. Le prêtre est souvent vu à travers le regard angoissé de l'enfant, p.ex. lorsque celui-ci se réveille à la suite d'un cauchemar et voit de Kern, penché sur son lit, comme « une grande ombre toute noire » au « regard fulgura[nt] dans la nuit» (MIRBEAU, 1890: 165). La mention de la cigarette allumée, qui accompagne souvent ce portrait tout en noir, y apporte une note rouge, le tout formant un ensemble chromatique diabolique, digne du Moine de Matthew Gregory Lewis, modèle du roman noir. Sauf que l'accent qui, dans ce dernier, était mis sur le «méchant», est ici déplacé sur sa victime, placée sur le devant de la scène dès le titre.

Anne-Laure Séveno (SÉVENO, 1997) prétend que Mirbeau renouvelle la représentation traditionnelle de l'enfant, aussi bien dans sa version romantique («enfant-ange ») que naturaliste («enfant-victime»), mais je pense qu'il la prolonge au contraire. Tout au plus complexifie-t-il le portrait de l'enfant, comme celui du pédophile, en y intégrant des traits ambigus (p.ex. pour Sébastien, le viol, malgré son horreur, reste lié à la révélation du plaisir érotique ; le Père de Kern n'est pas un monstre privé de tout remords), mais les deux personnages ne s'en inscrivent pas moins dans le système oppositionnel victime vs bourreau. Ce ne sont pas uniquement les événements de l'intrigue et ses portraits qui font du Père de Kern un persécuteur et de Sébastien - sa victime. La dimension victimologique est déjà présente dans le titre et, partant, dans l'étiquette du personnage qui combine les prénoms de deux saints - Sébastien et Roch. C'est surtout l'identification du protagoniste avec ce premier, dont le martyre par sagittation est l'un des motifs classiques de l'iconographie chrétienne, qui renforce le statut de victime du héros éponyme. Comme l'écrit Bérangère de Grandpré : « il est évident que Mirbeau reprend le lien étroit entre sexualité et violence qui existe dans les représentations picturales du saint »(GRANDPRÉ, 2006), basées sur la symbolique phallique des flèches. Saint Sébastien est un martyr mort par sagittation, le viol de Sébastien est aussi présenté dans le texte comme une sorte de martyre. Proche au début du roman de l'idéal rousseauiste de l'«enfant de la nature », cher aux romantiques, Sébastien se transforme ensuite progressivement, au contact du Père de Kern, en enfant martyrisé des naturalistes.

À côté du couple de personnages, c'est un espace-temps particulier qui fait partie de la matrice romanesque de la pédophilie, en renouant également avec le modèle du roman noir. Comme le note Gabriel Thoveron : «Le décor (...) est un élément essentiel du genre gothique » (THOVERON, 1996 : 134) qui a son espace typique, composé de lieux lugubres et mystérieux, cloîtres, souterrains, cachots, etc., ainsi que ses moments temporels ou atmosphériques privilégiés, comme la nuit ou l'orage. La scénographie de l'épisode du viol de Sébastien par le Père de Kern rentre dans ce schéma générique et, de ce fait, est lourde de symbolisme. Tout se passe un soir d'orage et le paysage que le Père de Kern contemple par la fenêtre du dortoir où il vient chercher Sébastien est des plus maléfiques : « (...) la nuit tourmentée, le ciel houleux où chevauchaient d'énormes vagues sombres, que la lune illuminait (...) d'éclatantes lueurs métalliques » (MIRBEAU, 1890 : 178). L'ecclésiastique entraîne ensuite son élève à sa suite dans un endroit reculé du collège où sera commis le viol. Au niveau symbolique, le parcours qu'ils suivent pourrait être qualifié de descente infernale. Le paysage orageux, le décor 
labyrinthique, le trajet descendant, les images de la claustration s'ajoutent au couple formé du bourreau et de sa victime et à l'effroi de cette dernière, pour former un ensemble correspondant parfaitement à l'imaginaire spatio-temporel du roman noir.

La matrice romanesque de la pédophilie se constitue également par un certain nombre de choix narratifs, notamment au niveau de la focalisation. Dans la scène précitée, Sébastien joue le rôle de focalisateur et l'on peut considérer que c'est sa peur intense qui, projetée sur le décor, rend celui-ci effrayant. Le choix du focalisateur est donc ici lourd de conséquences : en embrassant la perspective d'une victime terrifiée et donnant aux lecteurs et lectrices accès à ses pensées, le narrateur disqualifie du même coup le prêtre pédophile. Le discours intérieur du garçon, intégré à la narration à l'aide du style indirect libre, confère au récit une tonalité fortement inquiétante, comme dans cette série d'interrogations sur le comportement du prêtre : «Que préparait-il ? Quel supplice ? Quelle torture ?... Quelle mort ?» (MIRBEAU, 1890 : 180). Même lorsque le monologue narrativisé cède la place au psycho-récit et que le narrateur résume les pensées ou perceptions de Sébastien, le récit se transforme en une énumération d'horreurs : «Il crut entrevoir de terrifiantes faces d'assassins, des mains étrangleuses, des couteaux levés. (...) Il ne percevait plus qu'un bruit sourd, (...) sanglotant (...) de plaintes étouffées... » (MIRBEAU, 1890 : 180). Dans ce fragment, la terreur du garçon est tellement grande qu'elle provoque des hallucinations et ce n'est plus seulement le choix du focalisateur, mais aussi celui de l'objet focalisé (cf. BAL, 1997 : 149; GENETTE, 1983 : 51) - dans ce cas, un objet imaginaire généré par la peur - qui permet de renforcer la condamnation du prêtre pédophile en présentant les séquelles psychiques que ses agissements provoquent chez l'enfant. Le texte confine ainsi au fantastique, en l'occurrence d'origine psychique, autre trait qu'il partage avec le roman noir. Si le narrateur reste constamment très attentif aux mouvements intérieurs du personnage, c'est surtout pour en tirer des accents pathétiques, dus au rendu des émotions et impressions négatives qui ne cessent de le traverser. La narration est ainsi construite à l'image du psychisme du héros. Une constellation thématique en lien avec le sujet pédophile se met en place dans les fragments focalisés, notamment à travers le lexique employé par le narrateur, qui reprend souvent des termes du personnage (et même des métaphores entières), dominé par l'angoisse et le remords, à la suite de quoi la pédophilie se trouve associée notamment à la tentation diabolique, à la perdition, à la damnation, au martyre, à l'empoisonnement, à la corruption ou au « contre-nature».

La matrice romanesque de la pédophilie présuppose aussi un type particulier de narrateur. Si le rôle du personnage dans la conduite de la narration est primordial, ce n'est pas lui, mais un narrateur hétérodiégétique qui domine le récit et assume la fonction idéologique (axiologique). S'il adopte la perspective de Sébastien dans les passages focalisés, il ne se prive pas de manifester, à de nombreuses reprises, sa supériorité par des commentaires qui rompent cette «restriction de champ », p.ex. il souligne la perfidie du Père de Kern (cf. MiRBEAU, 1890 : 229) avant que Sébastien ne s'en rende compte (cf. MiRBEAU, 1890 : 241). Souvent, il lui arrive de formuler clairement ce qui apparait tout d'abord sous forme de pressentiments vagues du personnage. Son discours est - 138 - 
aussi parsemé de vérités générales dans lesquelles il entend transmettre un savoir p.ex. sur les lois de la vie en société ou l'éducation religieuse. Il est vrai qu'il existe aussi un deuxième narrateur dans ce roman : c'est Sébastien lui-même dans les fragments de son journal intégrés à la narration. En ce sens, le texte de Mirbeau diffère profondément des textes journalistiques consacrés à la pédophilie qui, à l'époque et jusqu'à une date relativement récente, si l'on en croit les analyses d'Anne-Claude Ambroise-Rendu (AMBROISE-RENDU, 2003 : 40), n'intégraient pas la voix des enfants dans leur dispositif énonciatif. Mais même le journal intime de Sébastien est récupéré par le narrateur à travers son commentaire, comme s'il devait toujours décider du sens en dernière instance. Quand le narrateur adopte la perspective du Père de Kern, le monologue narrativisé de celui-ci alterne avec des passages de psycho-récit où les pensées du prêtre sont clairement évaluées (cf. MIRBEAU, 1890 : 187-188). Il s'agit donc d'un narrateur fortement normatif qui est clairement au service du projet idéologique de l'auteur. Joanna EkiertZastawny a remarqué que : «(...) dans les romans de Mirbeau, la présence du narrateur se fait beaucoup sentir et (...) il est difficile d'opérer la distinction (...) entre l'écrivain et le narrateur » (EKIERT-ZASTAWNY, 2001). Dans Sébastien Roch aussi, le narrateur sert clairement de relais au système de valeurs de l'auteur qui écrit son livre dans une visée argumentative, sinon pamphlétaire, en se posant l'objectif de dénoncer l'éducation religieuse et présentant la pédophilie comme l'un des dangers de ce système éducatif.

La matrice romanesque de la pédophilie définit aussi bien ce qui est dit, par qui et comment, que ce qui reste tu, elle présuppose donc une organisation diégétique et narrative particulière. Présentée notamment à l'aide d'un réseau d'analepses et de prolepses, l'intrigue de Sébastien Roch trouve son point culminant dans le viol du héros éponyme par le Père de Kern, pour retracer ensuite les conséquences néfastes de cette violence sexuelle. Le mode de présentation de l'épisode du viol est à ce propos caractéristique. Toute une série de signaux funestes à valeur proleptique préparent cette scène. Le projet du père de Sébastien d'envoyer son fils dans un collège de jésuites est dès le début présenté, dans une formulation anticipatoire, comme ce qui va «écraser son fils 》 (MIRBEAU, 1890 : 10 ), et M. Roch en parle à Sébastien comme d' « une heure grave de [s]a vie... une heure décisive... » (MIRBEAU, $1890: 16$ ). D'une manière significative, la scène du viol de Sébastien elle-même est traitée par une ellipse, comme s'il s'agissait de l'irreprésentable. L'on pourrait dire, en raisonnant en termes psychanalytiques, que cette ellipse est celle d'un traumatisme tellement fort qu'il fait l'objet d'un refoulement. Ce traitement narratif de l'épisode converge aussi parfaitement avec l'attitude des journalistes de l'époque envers les affaires d'attentats à la pudeur. Dans la presse également, comme le note Anne-Claude Ambroise-Rendu, «L'événement n'est pas décrit, les journaux se contentent de signaler et de qualifier, pour les condamner, des actes qu'ils se refusent au fond à relater » (AMBRoISE-RENDU, 2003 : 33). Paradoxalement, ce blanc au cœur même du texte de Mirbeau attire l'attention sur l'événement qui est ainsi passé sous silence. Une ligne de points de suspension signale l'ellipse, après quoi c'est la réaction du garçon à ce qui vient de se passer qui est racontée, à nouveau en focalisation interne. Le blanc elliptique y reçoit une justification psychologique, le viol étant - 139 - 
censé avoir créé un vide psychologique et Sébastien étant caractérisé comme quelqu'un qui se retrouve "sans pensée» (MIRBEAU, 1890: 185). Quand un changement de focalisateur survient pour nous donner accès à la perspective du Père de Kern, c'est pour que ce dernier confirme que Sébastien est en proie à une « commotion cérébrale » (MIRBEAU, 1890: 187). Le traitement elliptique de la scène centrale est ensuite compensé par la représentation d'hallucinations et de rêves attribués au personnage, ses pensées marquées par l'horreur fonctionnant aussi comme révélatrices de la force du traumatisme subi.

C'est de la conjonction des éléments caractérisés ci-dessus, à savoir du couple victime-bourreau, d'un espace-temps construit conformément à l'imaginaire du roman noir, de l'attribution à l'enfant-victime du rôle de focalisateur, du normativisme du narrateur et du traitement elliptique de l'épisode sexuel, que résulte la matrice romanesque de la pédophilie. Si j'en juge par mon expérience d'usager des médias (et l'on connaît l'extraordinaire médiatisation des affaires de pédophilie à l'époque actuelle), cette matrice continue à influencer les discours contemporains sur la sexualité intergénérationnelle. Des spécialistes de l'analyse $\mathrm{du}$ discours médiatique pourraient vérifier cette affirmation par un examen systématique des sources contemporaines. À mon sens en tout cas, les conclusions de l'article susmentionné d'Anne-Claude Ambroise-Rendu sont compatibles avec l'optique que je défends. Mon objectif n'est toutefois pas de traquer la présence de la matrice romanesque de la pédophilie dans les médias d'aujourd'hui, mais de montrer que la littérature contemporaine offre une alternative à ce modèle discursif, ce à quoi sera consacrée la deuxième partie de mon article.

\section{Stratégies de déconstruction de la matrice romanesque de la pédophilie dans L'Agneau chaste}

\subsection{Stratégie éditoriale et critique}

Quiconque veut aborder aujourd'hui le sujet de la sexualité intergénérationnelle doit tenir compte d'un contexte socio-discursif dans lequel la matrice romanesque de la pédophilie joue un rôle important, sinon déterminant. Le dispositif péritextuel de la réédition de L'Agneau chaste au format de poche aux éditions de La Différence en 2002 apporte un éclairage intéressant à la question de savoir comment l'éditeur de Franck Varjac et l'une des critiques de son livre s'y prennent pour rendre acceptable la déconstruction de la matrice susmentionnée, effectuée par l'auteur. La quatrième de couverture porte trace du contexte sociodiscursif dans lequel l'oeuvre a été publiée et de ses conséquences pour sa réception. Le «péritexte éditorial» (GENETTE, 2002: 21) de la quatrième de couverture de la réédition résume en effet brièvement l'accueil de la première édition du livre : «Accueilli par le silence d'une presse tétanisée par le sujet - à l'exception, notable, du Monde -, L'Agneau chaste a néanmoins suscité de grands enthousiasmes et des adhésions inattendues ». Loin de tout misérabilisme éditorial, cette phrase essaie de tirer profit d'une réception critique apparemment limitée, pour à la fois poser le livre de Franck Varjac comme transgressif - sans toutefois préciser en quoi consisterait la transgression -, et construire l'image lectorale des happy few, capables de l'apprécier. Sont ensuite cités des propos de Josyane 
Savigneau du Monde, faisant visiblement partie de ce lectorat choisi et définissant plus clairement le sujet du roman :

« Il fallait sans doute du courage pour choisir comme sujet d'un premier roman une histoire d'amour entre un jeune garçon et un adulte en un temps où la juste dénonciation de la pédophilie, des contraintes inadmissibles imposées par des adultes à des enfants, a tout recouvert, y compris les ambiguittés de l'adolescence et le mystère de certaines rencontres improbables, de certaines initiations désirées et inoubliables. Il fallait aussi du talent pour réussir (...) un récit sur ce thème. Franck Varjac possède ce courage et ce talent. »

Ces propos de la fameuse critique du Monde témoignent de la négociation avec le contexte socio-discursif qui s'impose non seulement aux auteur et éditeur de L'Agneau chaste, mais également à la critique voulant aborder le livre. En effet, avant de louer les qualités du roman, la journaliste se sent visiblement obligée d'adhérer aux discours de dénonciation de la pédophilie. En prenant en compte l'état du discours social sur la question, elle construit aussi une certaine image de l'auteur de L'Agneau chaste, celle d'un écrivain non seulement talentueux, mais aussi courageux, car ayant osé s'attaquer à un thème tabou. Sous la plume de Josyane Savigneau, Franck Varjac rejoint ainsi la galerie des auteurs transgressifs, ce qui est bien sûr un trait élogieux, si l'on se rappelle qu'aussi bien les romantiques et le mouvement réaliste au XIX ${ }^{\mathrm{e}}$ siècle que les diverses avant-gardes au $\mathrm{XX}^{\mathrm{e}}$ ont érigé la transgression, qu'elle soit thématique ou formelle, en grande qualité littéraire. Ces stratégies de l'éditeur et de la critique ne constituent pourtant qu'un prolongement de celles mises en œuvre par l'auteur du livre et déconstruisant la matrice romanesque de la pédophilie.

\subsection{Stratégie narrative et diégétique}

Comment Franck Varjac s'y prend-il pour raconter une histoire d'amour entre David, un garçon de treize ans, et Fabrice, un homme de trente-deux ans, à une époque - la nôtre - où la matrice romanesque de la pédophilie semble dominer dans les discours sur la sexualité intergénérationnelle ? Il conteste pratiquement tous les éléments de la matrice présente dans le roman de Mirbeau. Dès la première page du livre se dessine une stratégie narrative alternative. Si, dans Sébastien Roch, nous avions affaire à un narrateur hétérodiégétique, autoritaire et fortement normatif, dans L'Agneau chaste, l'auteur confie le récit à un hérosnarrateur : ce garçon de treize ans ou, plus exactement, l'homme qu'il est devenu. Le choix de la personne du narrateur et de sa position temporelle ont autant d'importance l'un que l'autre. Il s'agit d'un narrateur homodiégétique et d'une narration ultérieure, faite a posteriori. Celle-ci n'est donc pas vraiment menée par un enfant engagé dans une liaison pédophile, qu'on pourrait supposer manipulé par un adulte pervers, mais par un homme psychologiquement mûr et ayant pris de la distance par rapport à sa propre histoire. Si les événements ne sont pas racontés par un observateur extérieur, ils ne le sont pas non plus par un héros encore sous le coup de l'émotion. Un tel choix narratif semble dicté par la volonté de conférer au récit une plus grande fiabilité. Si la narration de David est empreinte d'une émotion, c'est la nostalgie d'une époque de bonheur révolue qui l'imprègne. Bien qu'il s'agisse d'un narrateur homodiégétique, celui-ci garde une neutralité assez 
poussée, évite de juger les autres personnages et prononce très peu de commentaires à valeur normative, ce qui contraste fortement avec le normativisme du narrateur de Mirbeau. Dans L'Agneau chaste, l'attitude du héros-narrateur envers les autres protagonistes se laisse déduire de certains rapprochements ironiques, p.ex. lorsqu'il nous fournit des informations sur la moralité « exemplaire » de sa famille - celle qui l'a jugé sans pitié en apprenant sa liaison avec Fabrice - en effet, il apprend qu'à la même époque, sa mère vivait une aventure avec son moniteur de plongée, et que son père et son frère ne cessent de changer de femme. Toutefois, David ne les juge pas : il se contente de réagir à ces révélations de sa sœur par un grand rire qui signale le décalage entre le moralisme dont ses proches ont fait preuve à son égard et leur propre comportement sexuel dans lequel ils s'autorisent beaucoup plus de liberté.

Le couple de personnages que nous avons rencontré dans Sébastien Roch, composé d'une victime et d'un bourreau, disparaît de L'Agneau chaste. Le rapport de forces entre les personnages est inversé : chez Mirbeau, il était asymétrique et profondément inégalitaire, jouant en défaveur du personnage de l'enfant. Le hérosnarrateur de Varjac interprète sa relation à l'adulte tout à fait différemment : « Je me sens fort, d'une force et d'un pouvoir étonnants. Il me semble que Fabrice m'appartient plus que je ne lui appartiens (...) je le sens frémir sous mes doigts... » (VARJAC, 2002: 58). Dans la description de leur première scène d'amour, le héros-narrateur met également l'accent sur la timidité et la peur de son partenaire et sur la force de son propre désir. L'on est très loin de la figure de l'enfantvictime vulnérable et impuissante. Qui plus est, dès la première page, David fait preuve d'une attitude positive par rapport à l'épisode raconté : ce n'est pas la voix d'une personne traumatisée par la rencontre avec un «prédateur sexuel » que nous entendons ; au contraire, il qualifie sa liaison juvénile avec Fabrice de «moments de bonheur infini » et de «moments d'amour pur» (VARJAC, 2002: 7). Cette insistance sur la pureté se situe clairement dans le prolongement du titre du roman. Dans Sébastien Roch, la voix de l'enfant était utilisée afin de diaboliser le pédophile, Franck Varjac l'utilise pour dédramatiser la sexualité intergénérationnelle. Non seulement son narrateur adopte une tonalité empreinte de nostalgie par rapport à l'aventure vécue avec son amant adulte, mais aussi, il intègre à la narration le discours contemporain sur la pédophilie, qui prolonge la matrice mise en place dans Sébastien Roch, de manière à le mettre à distance. En effet, après s'être déjà engagé dans la relation avec Fabrice, il résume ainsi les réactions de ses camarades à l'histoire d'un responsable de colonie de vacances renvoyé pour pédophilie : «(...) rires gras, mépris, violence aussi. Une meute aux dents neuves et meurtrières » (VARJAC, 2002: 48). À travers cette image inquiétante, l'on voit que ce qui suscite la crainte du héros-narrateur, c'est l'attitude de son entourage envers la sexualité intergénérationnelle. Un peu plus loin, il essaye de désamorcer le discours sur le danger social qu'elle représenterait, en se demandant: «À qui cela pourrait-il nuire ?» (VARJAC, 2002: 51). À un autre moment (cf. VARJAC, $2002: 58-59$ ), c'est l'attitude hostile de ses camarades à l'égard de l'homosexualité qui inquiète David. Et lorsqu'il essaie d'imaginer la réaction de ses proches à une éventuelle révélation de sa liaison avec Fabrice (cf. VARJAC, 2002: 44-45), il craint le pire (ses prédictions se réaliseront d'ailleurs). 
La source des émotions négatives qui le traversent, ce n'est donc pas sa relation avec un adulte, mais l'hostilité de son entourage envers tout non-conformisme sexuel.

Enfin, au niveau diégétique, les rôles dans l'histoire sont aussi distribués d'une manière qui tranche avec la matrice romanesque de la pédophilie : c'est Fabrice, le «pédophile » qui y a le beau rôle. Le schéma diégétique est celui d'une histoire d'amour romantique, d'un sentiment partagé, contrarié par les conventions sociales. Accompagné d'une série de présages (le mot est explicitement employé par le narrateur ; cf. VARJAC, 2002: 45), introduisant l'idée d'une fatalité qui pèserait sur les amants, ce scénario est propice à l'éveil de l'empathie et de la sympathie envers les personnages. Ce n'est pas le séducteur adulte qui apparaît comme oppresseur mais ceux qui séparent les amants : le gardien du gymnase où ils se rencontrent, qui surprend une scène d'amour entre eux, et la famille du narrateur qui réagit à cette révélation fortuite de leur relation d'une manière violente. En effet, dans L'Agneau chaste, la violence psychologique et physique est liée non pas au rapport entre l'adolescent et l'adulte, mais à la réaction de la famille et de l'entourage à son dévoilement : le narrateur est alors brutalisé par son père, insulté et enfermé dans sa chambre, ses proches refusent de lui adresser la parole et décident enfin de l'envoyer dans un pensionnat pour qu'il y finisse sa scolarité. La suite de sa vie est placée sous le signe de la rupture des liens familiaux : sa famille le renie, il lui est interdit de participer aux funérailles de ses grands-parents, seule sa sœur lui rend quelquefois visite et toute cette situation est résumée par le narrateur à travers la formule de l'«oubli assassin de [s]es proches» (VARJAC, 2002: 7). Ce développement de l'intrigue converge avec certaines recherches sur la pédophilie, signalées par le philosophe Igor Primoratz dans son livre Ethics and Sex, qui distinguent les relations pédophiles basées sur la violence et provoquant de graves traumatismes psychologiques des rapports consentis et non violents qui semblent ne pas entrainer de séquelles (cf. PRIMORATZ, 2012 : 195-198). Ces études montrent notamment que la source du traumatisme se situe souvent non pas dans la liaison pédophile elle-même, mais dans la réaction de l'entourage à sa découverte, ce qu'illustre justement le cas du narrateur de L'Agneau chaste.

\subsection{Stratégie culturelle et symbolique}

L'auteur de L'Agneau chaste déconstruit la matrice romanesque de la pédophilie en mettant à contribution non seulement une stratégie narrative et une configuration diégétique particulières, mais également des références culturelles et une construction symbolique de l'espace. Ces deux derniers éléments sont liés. L'on sait, grâce à des études historiques et anthropologiques, que les relations sexuelles entre hommes et garçons, ont eu, dans de nombreuses cultures, une valeur initiatique. Or, l'histoire de David et Fabrice est clairement inscrite dans un cadre spatio-temporel initiatique. Symboliquement, c'est la présence de l'élément aquatique et maritime qui structure l'intrigue. Dans la première scène, le hérosnarrateur, entouré de sa famille, fête son $13^{\mathrm{e}}$ anniversaire sur une plage. Fabrice, son futur amant, arrive par la mer, sur un bateau. Quant on se rappelle que la mer symbolise « la dynamique » et « un état transitoire » (CHEVALIER ; GHEERBRANT, 
1982: 623) et l'eau «l'infinité des possibles» (CHEVALIER; GHEERBRANT, 1982 : 374) et qu'on y ajoute l'âge de David, au seuil de la puberté, sa présence au bord de l'eau semble suggérer qu'il se trouve à un moment charnière de sa vie. Dans la même scène, Fabrice commente d'ailleurs le physique de David, affirmant qu'il paraît plus âgé qu'il n'est en réalité. Il semble donc prêt au passage à l'âge adulte. L'arrivée de Fabrice sur la plage confirme la dimension initiatique du texte. D'une manière significative, le bateau sur lequel il arrive est un zodiac, ajoutant «le symbolisme de l'évolution biologique et psychologique» (CHEVALIER; GHEERBRANT, 1982: 1040) du zodiaque au symbolisme aquatique déjà évoqué. La forte présence de l'eau dans la première partie du roman renvoie bien évidemment aussi à la pureté, en résonnant avec le titre du livre.

Mais la première rencontre sur la plage annonce seulement l'initiation sexuelle qui aura lieu plus tard, sur une autre plage, à l'occasion d'une partie de pêche à laquelle Fabrice invite David. Le contact physique entre l'homme et le garçon y est présenté comme désiré par les deux partenaires et cette partie de pêche initiatique s'inscrit parfaitement dans une configuration qui, tant symboliquement que psychologiquement, est à l'opposé de celle présente dans Sébastien Roch. La luminosité des scènes de L'Agneau chaste, où l'espace est baigné de soleil, fait partie de cette opposition. À ce propos, l'illustration de couverture de l'édition que j'ai utilisée pour cette analyse est particulièrement bien choisie: il s'agit d'une reproduction du tableau Noonday Heat de Henry Scott Tuke, représentant deux adolescent nus, sur une plage noyée de lumière. L'omniprésence de la lumière dans les scènes des premières rencontres entre David et Fabrice contraste avec l'obscurité dans laquelle est le plus souvent plongé le décor dans Sébastien Roch. Elle pourrait aussi emblématiser le traitement de la sexualité dans le roman de Varjac. Loin de faire l'objet d'une ellipse, comme chez Mirbeau, le contact érotique entre l'homme et le garçon est décrit tout à fait ouvertement et présenté comme source de plaisir pour les deux partenaires. Il ne provoque pas de traumatisme, mais au contraire, est ressenti par le héros-narrateur comme l'expérience érotique la plus gratifiante de sa vie. L'image idyllique d'une nature lumineuse entre en résonance avec le ressenti des personnages. Dans la suite du roman, les rencontres entre les amants se passent dans un gymnase, lieu qui introduit une référence à la Grèce antique et au modèle pédérastique, cette relation pédagogique entre un éraste et un éromène, ce qui confirme encore une fois la dimension initiatique dont ce texte dote la sexualité intergénérationnelle.

\section{Conclusion}

À la suite d'une évolution socio-sémantique, l'étiquette «pédophilie » a fini par recouvrir des comportements sexuels très divers qui, comme le signale Igor Primoratz, ne sont pas nécessairement synonymes de violence, d'abus et de traumatisme. Si le philosophe nous invite à une réflexion plus rationnelle et plus nuancée sur le phénomène de la sexualité intergénérationnelle, Franck Varjac semble faire de même par l'intermédiaire de son roman. Aujourd'hui, la matrice romanesque de la pédophilie, construite dans Sébastien Roch et issue du roman noir, domine dans les discours tenus sur ce sujet, ce dont le péritexte éditorial du 
livre de Varjac porte trace. Il serait possible d'attribuer à sa déconstruction par l'auteur de L'Agneau chaste une valeur éthique, allant dans le même sens que les avertissements de Primoratz. Toutefois, si l'on voulait vraiment s'inscrire dans la logique proposée par ce roman, il faudrait le soustraire à toute considération morale car en proposant une alternative au traitement habituel de la sexualité intergénérationnelle, Franck Varjac semble essayer de la situer « au-delà du bien et du mal».

\section{BIBLIOGRAPHIE}

AmbRoISE-RENDU Anne-Claude (2003), Un siècle de pédophilie dans la presse (1880-2000) : accusation, plaidoirie, condamnation, Le Temps des médias, $\mathrm{n}^{\mathrm{o}} 1$, p. 31-41.

BAL Mieke (1997), Narratology. Introduction to the Theory of Narrative, TorontoBuffalo-London, University of Toronto Press.

BEAUMARChaIS Jean-Pierre de ; COUTY Daniel, ReY Alain (1984), Dictionnaire des littératures de langue française, Paris, Bordas.

CHAUMON Franck (2005), Le pédophile, notre frère, in : PALACiOS Marcela (dir.), Enfants, sexe innocent? Soupçons et tabous, Autrement, coll. Mutations, $\mathrm{n}^{\mathrm{o}} 234$, p. $100-114$.

CheVAlier Jean ; GHeERBRANT Alain (1982), Dictionnaire des symboles, Paris, Robert Laffont/Jupiter.

Courouve Claude (1985), Vocabulaire de l'homosexualité masculine, Paris, Payot.

EKIERT-ZASTAWNY Joanna (2001), Quelques remarques sur le narrateur dans les romans d'Octave Mirbeau, [http://mirbeau.asso.fr/darticlesfrancais/EkiertZastawny-narrateur.pdf] (consulté le 14 février 2015).

FERRON Laurent (2001), Le viol de Sébastien Roch : l'Église devant les violences sexuelles, (consulté le 3 avril 2015).

FouCAUlT Michel (1976), La Volonté de savoir, Paris, Gallimard.

GENETTE Gérard (1983), Nouveaux discours du récit, Paris, Seuil.

GENETTE Gérard (2002), Seuils, Paris, Seuil.

GRANDPRÉ Bérangère de (2006), La figure de Saint Sébastien de Mirbeau à Trakl, [http://mirbeau.asso.fr/darticlesfrancais/Grandpre-Sebastien.pdf] (consulté le 15 février 2015).

HeKMa Gert (1999), The Gay World: 1980 to the Present, in : ALDRICH Robert (éd.), Gay Life and Culture: A World History, London, Thames \& Hudson, p. 333-363.

Michel Pierre (2005), Octave Mirbeau et le roman, Angers, Société Octave Mirbeau.

MiRBEAU Octave (1890), Sébastien Roch. Roman de mœeurs, Paris, G. Charpentier et $\mathrm{C}^{\text {ie }}$, Editeurs.

PRIMORATZ Igor (2012), Filozofia seksu, trad. Joanna Klimczyk, Warszawa, Wydawnictwo Naukowe PWN. 
SÉVENo Anne-Laure (1997), L'enfance dans les 'romans autobiographiques' d'Octave Mirbeau: démythification et démystification, <http://mirbeau.asso.fr/darticlesfrancais/Seveno-enfance.pdf> (consulté le 8 février 2015).

THOVERON Gabriel (1996), Deux siècles de paralittérature. Lecture, sociologie, histoire, Liège, Éditions du CEFAL.

VARJAC Franck (2002), L'Agneau chaste, Paris, La Différence.

WOLF Nelly (2014), Proses du monde. Les enjeux sociaux des styles littéraires, Villeneuve d'Ascq, Presses Universitaires du Septentrion.

ŻABSKI Tadeusz (2006), Stownik literatury popularnej, Wrocław, Wydawnictwo Uniwersytetu Wrocławskiego. 